\title{
Augmented Reality Technology As A Delivery Mechanism For Psychological Intervention In Adolescents With Asthma: A Randomised Controlled Feasibility Trial Protocol
}

Kelsey Sharrad ( $\sim$ kelsey.sharrad@unisa.edu.au )

University of South Australia https://orcid.org/0000-0002-0301-7161

Caitlin Martini

University of South Australia

Zoe Kopsaftis

University of Adelaide

Andrew Tai

Women's and Children's Hospital

Nicola Spurrier

Government of South Australia, Adelaide

Ross Smith

Australian Research Centre for Interactive and Virtual Environments

Adrian Esterman

University of South Australia

lan Gwilt

University of South Australia

Debra Sandford

University of Adelaide

Kristin Carson-Chahhoud

University of South Australia

\section{Study Protocol}

Keywords: Asthma, augmented reality, psychological intervention, psychological distress, adolescents

Posted Date: December 28th, 2021

DOI: https://doi.org/10.21203/rs.3.rs-1122342/v1

License: (9) This work is licensed under a Creative Commons Attribution 4.0 International License.

Read Full License 
Page 2/14 


\section{Abstract}

Background: Australia has one of the highest rates of asthma prevalence worldwide, with almost one in 10 children affected. The mental health and wellbeing of asthmatic children is reported to be significantly more impacted than non-asthmatic peers; affecting both asthma management and their overall quality of life. The relationship between asthma and psychological distress is likely bi-directional, therefore requiring an intervention that addresses both psychological and physiological factors. Technology-based psychological interventions provide a potential solution that may increase engagement with treatment amongst adolescents. In particular, augmented reality (AR) is a novel technology that can be tailored to individual populations and has been proven effective in the management of other conditions. No evidence exists currently on the feasibility of AR in the management of psychological wellbeing within the asthmatic community.

Methods: An 80-page workbook has been developed based on best-practice asthma guidelines and through consultation with field experts in Psychology and Paediatric Respiratory Medicine, AR tools have been developed following a needs assessment, and a bespoke smartphone app has been developed by Portal Australia. Forty $(n=40)$ young people aged 13-17 years with persistent asthma will be recruited from the Respiratory Department at the Women's and Children's Hospital. Participants will be required to download a smartphone application (YOLO de-stress) and will be encouraged to use the app for a month. Participants will be required to complete the Youth Asthma-related Anxiety Scale, Paediatric Asthma Quality of Life Questionnaire and the Asthma Control Questionnaire at baseline and one-month postintervention. Data analysis will be descriptive, with counts and percentages for categorical data, and means and standard deviations for continuous data compared between baseline and follow-up. For continuous outcome variables mixed effects linear models will be used. For other outcome measures mixed effects generalized linear models will be used.

Discussion: This study will explore the feasibility of AR tools to aid delivery of psychological intervention to manage symptoms of elevated psychological distress among young people with asthma, and inform development of a fully powered RCT.

Trial registration: This project was prospectively registered with the Australian New Zealand Clinical Trials Registry (ANZCTR): ACTRN12620001109998

\section{Background}

\section{Asthma in adolescence}

Australia has one of the highest asthma prevalence rates in the world (1), with approximately 2.7 million adults living with the condition (2). Moreover, a 2017-18 review reports almost one in ten Australian children under 14 suffer from this chronic and debilitating condition (3). The health and mental wellbeing of young people with asthma is reportedly worse than their non-asthmatic peers, particularly anxiety and depressive symptoms. Two separate meta-analyses looking at the prevalence of anxiety disorders and 
depression/anxiety symptoms, reported that those with asthma display a three times increased prevalence (4) and a one in four chance of experiencing symptoms (5) respectively. Furthermore, a 2014 survey of Australian asthmatic young people (12 to 25 years), found $50 \%$ experienced symptoms of elevated psychological distress; double the general population rate (6).

\section{Asthma and psychological distress in adolescence}

The increased psychological distress experienced by young people with asthma is likely compounded by a range of social, psychological and developmental challenges traditionally experienced by adolescents $(7,8)$. These challenges may include social isolation, restricted independence and selfconsciousness (9), which may all combine to decrease quality of life (10), increase functional impairment (11), and incur higher preventable hospital visits compared to non-asthmatic peers (12). However, it is likely the relationship is bidirectional between asthma and psychological distress (13) as psychological factors can exacerbate asthma symptoms (14-16) and potentially decrease medication adherence and proper asthma management (13). Utilising psychological interventions may assist in managing psychological distress within young people, therefore assisting in asthma management (17). One example of said interventions are 'behaviour-based therapies'. Acceptance and Commitment Therapy (ACT) is a mindfulness-based behaviour therapy that focuses on how positives and negative thoughts and events are part of life, and encourages acceptance of thoughts and feelings (18). The goal of ACT is to build psychological flexibility to assist in building and managing a life that aligns with ones values (18). In the area of young people with symptoms of mental health conditions, an adolescent case study of a female with anorexia nervosa reported successful adoption of ACT in practice, suggesting that despite her thoughts about body dissatisfaction, she was able to adapt her behaviour and adopt healthier eating practices (19). Furthermore, ACT has been evidenced to improve symptoms in clinically depressed individuals both immediately and 3 months post-therapy (20), and assist in the management of chronic paediatric pain as part of a behavioural medicine approach (21). Within the asthma community, ACT has been integrated into parental education and shown to be effective in decreasing parental anxiety and stress of those with children aged 3-12 (22), and improve the psychological wellbeing of adult asthmatic patients (23). While there has been evidence of ACT success with parental and adult populations, more research is needed in adolescent populations.

\section{Technology for health interventions}

The 2012-16 National Youth Mental Health Survey identified the internet as a leading source of information for youth (9), with self-help and self-reliance preferred over seeking help from professionals or family and friends (24). Unguided self-help however, varies in effectiveness due to the vast range, quality of sources available and varying retention rates (25).

To address said issues, authorities are seeking technology-based solutions to increase engagement amongst young people and tackle current barriers influencing care for both mental health $(9,10)$ and asthma management $(9,26)$. Technology-delivered psychological interventions in particular, have the means to be effective within mental health care as a low-cost, low-burden option (9). These interventions 
increase access and quality of care (27), preserve anonymity, and provide an alternate option to those in remote and rural locations (28). A randomised control trial combining an online ACT intervention for adults living with Fibromyalgia with their normal treatment regime increased pain acceptance significantly, which lead to a significant improvement in Fibromyalgia impact both post intervention and at 3-months follow up (29). Combining ACT interventions with normal treatments has additionally proven effective in targeting parents of, and children living with Autism Spectrum Disorder by decreasing parental stress, increasing child prosocial behaviours and decreasing hyperactive behaviours (30). Further research is needed into how best to make these online ACT interventions (similar to those aforementioned) into effective treatments for a range of other populations. One novel solution may be augmented reality (AR) technology.

\section{Augmented reality for health interventions}

AR technology superimposes digital information within the real world through a smartphone to create an interactive learning environment (31) that is able to be tailored to specific populations to promote increased user engagement (32). AR provides multiple other benefits; from improving accessibility of information and geographic reach due to its portability (33), to addressing low health literacy by utilising videos, graphics and animation rather than relying on written information $(26,31)$. AR has been evidenced to provide additional learning opportunities to assist in the education of school-aged children (34) and has been shown to be a feasible tool in assisting with the psychological management of phobias (35). Moreover, a 2017 pilot study reported AR as a helpful tool in education of correct inhaler techniques amongst asthmatic individuals and health professionals (36), however little evidence currently exists to support the use of AR as a delivery mechanism of psychological interventions for asthmatic individuals. To overcome previous limitations, this research will examine the feasibility of AR technology as a delivery mechanism for an ACT intervention, in order to manage symptoms of elevated psychological distress among young people with asthma.

\section{Methods/design}

\section{Aims}

Explore the feasibility of AR tools to aid the delivery of ACT to manage symptoms of elevated psychological distress among young people with asthma, aged 13 to 17 years. This will be achieved through:

1.) Development of a paper-based workbook and $A R$ resources for delivery via bespoke smartphone application. These tools have been developed following a needs assessment of the biopsychosocial needs of adolescents with asthma (37), and refined based on qualitative feedback obtained in Stage $2 a(38)$.

2.) Evaluation of the practicality and feasibility of these ACT resources for use by youth (13-17 years), their guardians and health professionals via: 
a.) Qualitative research which has been completed and published elsewhere (38).

b.) Randomised controlled feasibility study of AR ACT + self-help smartphone application (YOLO.destress) versus self-help smartphone application alone for treatment of symptoms of anxiety among young people with asthma, with one-month follow-up.

\section{Hypothesis}

Children with asthma using AR resources plus self-help smartphone app compared to children using the self-help app alone by 1-month will have:

i. Increased use (number of logins, total time and adherence data) of smartphone resource measured through smartphone access logs (primary outcome)

\section{Secondary outcomes:}

i. Reduced anxiety symptoms measured by the Youth Asthma-related Anxiety Scale (YAAS)

ii. Improved quality of life measured by the Paediatric Asthma Quality of Life Questionnaire (PAQLQ)

\section{Project Design}

\section{Stage 1: Development of workbook, AR resources + self-monitoring smartphone app}

An 80-page workbook has been developed based on best-practice asthma guidelines and through consultation with field experts in Psychology and Paediatric Respiratory Medicine. Content includes educational information about asthma and/or psychological distress, and ACT strategies such as mindfulness activities (see appendix 1 for examples from workbook). When scanned through the YOLO_de.stress app on the participant's smartphone, printed resources will seemingly come to life with digital content via AR technology.

The AR tools have been developed following a needs assessment of the biopsychosocial needs of adolescents with asthma (37), and refined based on qualitative feedback obtained in Stage 2a (38). AR video content (around 1-minute duration) was filmed with a depth camera and 3608 digital photography using investigators from this proposal. Both real world settings and green screen backdrops were used to facilitate digital environments. AR resources have been custom-built by the investigator team using Unity software. The type of content filmed includes educational information about asthma and ACT strategies, as well as verbal and visual instruction on how to perform ACT techniques.

The self-help smartphone application has been developed by Portal Australia. It has been designed to facilitate navigation to credible online self-help websites and track self-monitoring of psychological symptoms. It will also be the access platform for AR tools. Daily symptom recordings will be displayed in four visual charts (pie, line, bar and odometer graphs) for real-time feedback on their health management. Data from the app will be forwarded electronically to investigators and used to track app usage. 


\section{Stage 2a: Evaluation of AR resources - Qualitative research}

This research has been undertaken previously (HREC/18/WCHN/172), and data has been published elsewhere (38).

\section{Stage 2b: Evaluation of AR resources - Randomised controlled feasibility study.}

Aim: To determine the feasibility of undertaking a fully-powered randomised controlled trial, through a feasibility pilot study.

Participants: Forty ( $n=40)$ young people (13 to 17 years) with persistent asthma will be recruited from the Respiratory Department at the Women's and Children's Hospital. Participants will be identified through Respiratory Department inpatient and outpatient lists at the Women's and Children's Hospital, and via flyers and radio/television news stories advertising the project. Hospital staff will provide potential participants with a copy of the participant information sheet and consent form for their review. Participants recruited through hospitals will only be approached by research staff once written consent has been provided to say that they are willing to discuss the project more with research staff, in order to undertake informed consenting procedures. Participants recruited via flyers and radio/television news stories will contact research staff to begin informed consenting procedures. As this is a feasibility study, a sample size calculation is not required. A sample of $n=40$ participants will be sufficient based on other similar studies $(39,40)$ to examine the primary outcome of AR smartphone resources (process measures) between the two groups.

\section{Inclusion criteria:}

Young people will be eligible for inclusion in this study if they: are aged between 13 and 17 years; have been formally diagnosed with asthma by a health professional; have experienced or are currently experiencing heightened symptoms of anxiety determined by self-report on the K10+ questionnaire; have access to a smartphone with the owner's permission to use it; are English speaking/able to understand written English.

\section{Exclusion criteria:}

Participants with an intellectual disability or cognitive impairment that would inhibit their ability to provide informed consent and participate in the project will be ineligible to participate.

Procedure: Hospital staff will provide potential participants with a copy of the participant information sheet and consent form for their review. Participants will only be approached by research staff once written consent has been provided to say that they are willing to discuss the project more with research staff, in order to undertake informed consenting procedures. Research staff will meet with potential participants to conduct eligibility screening and informed consenting procedures in meeting spaces in the respiratory department at Women's and Children's Hospital (WCH), in meeting spaces at the South Australia Health and Medical Research Institute (SAHMRI), or online via Zoom, at the participant's 
convenience. Randomisation was performed in Microsoft Excel using the "=Rand()" function. Forty participant ID numbers were listed in column A, the =RAND() function was then applied in column B. A filter function was added to both rows, whereafter column B was sorted from 'Largest to Smallest'. The first 20 numbers were allocated to Cohort \#1 (Control), the second lot of 20 numbers were allocated to Cohort \#2 (Intervention). This randomization process was conducted by a volunteer who was not involved in the recruitment, interview, or analysis process conducted in accordance with this project. The primary researchers (KS, KVCC) were blinded to the allocation of participant IDs to cohorts. Data analysts will also be blinded to group allocation.

Following consenting, screening, and randomisation processes, baseline assessments will be conducted, including completion of the Youth Asthma-related Anxiety Scale (YAAS), the Paediatric Asthma Quality of Life Questionnaire (PAQLQ), and the Asthma Control Questionnaire (ACQ). The smartphone app (YOLO de-stress) will be downloaded onto participant smartphones, and they will be provided with a tutorial by research staff. Intervention participants will also be provided with a copy of the workbook, and shown the AR tutorial. All participants will be asked to use the app for the one-month study period, during which time they will be presented with a booster session encouraging app usage through positive affirmation via push notifications at weekly intervals post-recruitment.

At 1-month post-intervention, research staff will again meet with participants in meeting spaces at WCH, SAHMRI, or online via Zoom. Follow-up assessments include completion of the YAAS, PAQLQ, ACQ.

\section{Data analysis}

Participant flow through the trial will be displayed using a CONSORT diagram. Analysis of the questionnaire data will be descriptive, with counts and percentages for categorical data, and means and standard deviations for continuous data compared between baseline and follow-up. Missing information from quantitative scales and surveys will be accounted for through random imputation. Drop-outs or loss to follow-up will be compared between study arms, and against those remaining in the trial. For continuous outcome variables mixed effects linear models will be used. For other outcome measures mixed effects generalized linear models will be used.

\section{Data management}

Participants will be de-identified, and will be provided ID numbers as opposed to names. De-identified data will not be stored in the same place as identified data (e.g. names, contact numbers). All data collected will be stored electronically in a password protected database on the secure University of South Australia network, while all hard copies will be stored in a locked filing cabinet in the South Australia Health and Medical Research Institute building. It will be stored for seven years in accordance with responsible research guidelines.

As the developers of the smartphone application, Portal Australia will have access to all the data in the system for the purpose of providing a working system, and exporting any data needed for analysis. They 
will not keep any data beyond the study. They will not have access to any identifying information about participants as that lives outside the system - participants will be registered to use the app by study staff, and will use an ID number to log on.

\section{Discussion}

This randomised controlled feasibility study will explore the feasibility of AR tools to aid the delivery of ACT to manage symptoms of elevated psychological distress among young people with asthma, aged 13 to 17 years. While evidence in other areas of healthcare and education is promising, little research has been conducted to support the use of AR as a delivery mechanism of psychological interventions for asthmatic adolescents.

Participants will receive a report once the project has been completed. Additionally, where appropriate, participants will receive a copy of any subsequent publications. Upon completion of the project, data will be synthesised for use in publications and conferences. Reports will be provided to participants and relevant consumer stakeholders, as well as funding bodies as per requirements. All ethical reporting procedures will be followed.

This study will provide essential pilot data to guide development of a grant submission for a fully powered RCT if shown to be feasible.

\section{List Of Abbreviations}

ACQ - Asthma Control Questionnaire

ACT - Acceptance and Commitment Therapies

AR - Augmented reality

IM - Intervention Mapping

PAQLQ - Paediatric Asthma Quality of Life Questionnaire

SAHMRI - South Australian Health and Medical Research Institute

WCH - Women's and Children's Hospital

YAAS - Youth Asthma-related Anxiety Scale

\section{Declarations}

\section{Ethics approval and consent to participate}


This project is conducted in accordance with the principles of the Declaration of Helsinki (41) and has received ethical approval by the Human Research Ethics Committee for the Women's and Children's Health Network (2021/HRE00055), and the University of South Australia Ethics Committee (Application ID: 204144).

\section{Consent for publication}

Not applicable

\section{Availability of data and materials}

All data collected will be stored electronically in a password protected database, while all hard copies will be stored in a locked filing cabinet in the South Australia Health and Medical Research Institute building.

\section{Competing interests}

The authors declare that they have no competing interests.

\section{Funding}

This project is supported by project funding received from the Channel 7 Children's Research Foundation (19/10693585), Early Career Fellowship funding received from NHMRC (GNT1141521), and project funding received from NHMRC (GNT1108903) by Associate Professor Carson-Chahhoud.

\section{Authors contributions}

$\mathrm{KCC}$ is overseeing all aspects of the project, from design to implementation to analysis and interpretation of data; AT provided expertise and knowledge of asthma, contributed to the development of AR resources, and will assist with recruitment from hospital sites; RS provided expertise for the development of AR technology; AE provided statistical analysis guidance; IG is responsible for creative design input for AR resources; DS provided expert clinical knowledge about mental health issues, and contributed to the development of AR resources; KJS is responsible for project management and manuscript production; all authors provided feedback on the draft, and approved the final manuscript.

\section{Acknowledgements}

Not applicable.

\section{References}

1. GINA. Global Strategy for Asthma Management and Prevention. Global Initiative for Asthma; 2018.

2. \#1 A. Asthma. Canberra: Australian Institute of Health and Welfare; 2020.

3. \#2 A. Australia's Children. Canberra: Australian Institute of Health and Welfare 2020. 
4. Dudeney J, Sharpe L, Jaffe A, Jones EB, Hunt C. Anxiety in youth with asthma: A meta-analysis. Pediatric Pulmonology. 2017;52(9):1121-9.

5. Lu Y, Mak K, van Bever HPS, Ng TP, Mak A, Ho RC. Prevalence of anxiety and depressive symptoms in adolescents with asthma: A meta-analysis and meta-regression. Pediatric Allergy and Immunology. 2012;23(8):707-15.

6. Blanchard M, Morris J, Birrell E, Stephens-Reicher J, Third A, Burns JJMY, et al. National young people and asthma survey: issues and opportunities in supporting the health and wellbeing of young people living with asthma. 2014.

7. Cohen R, Franco K, Motlow F, Reznik M, Ozuah PO. Perceptions and Attitudes of Adolescents with Asthma. Journal of Asthma. 2003;40(2):207-11.

8. de Benedictis D, Bush A. The Challenge of asthma in adolescence. Pediatric Pulmonology. 2007;42(8):683-92.

9. Mission Australia BDI. Youth mental health report: youth survey 2012-2016. Australia; 2017.

10. Goldney RD, Ruffin R, Fisher LJ, Wilson DH. Asthma symptoms associated with depression and lower quality of life: a population survey. The Medical journal of Australia. 2003;178(9):437-41.

11. Akinbami LJ, Schoendorf KC. Trends in childhood asthma: prevalence, health care utilization, and mortality. Pediatrics. 2002;110(2 Pt 1):315-22.

12. Calmes D, Leake BD, Carlisle DM. Adverse Asthma Outcomes Among Children Hospitalized With Asthma in California. Pediatrics. 1998;101(5):845.

13. Baiardini I, Sicuro F, Balbi F, Canonica GW, Braido F. Psychological aspects in asthma: do psychological factors affect asthma management? Asthma research and practice. 2015;1:7.

14. French TM, Alexander F. Psychogenic Factors in Bronchial Asthma. British medical journal. 1943;1(4291):416-.

15. Sandberg S, Paton JY, Ahola S, McCann DC, McGuinness D, Hillary CR, et al. The role of acute and chronic stress in asthma attacks in children. Lancet (London, England). 2000;356(9234):982-7.

16. Van Lieshout RJ, Macqueen G. Psychological factors in asthma. Allergy, asthma, and clinical immunology : official journal of the Canadian Society of Allergy and Clinical Immunology. 2008;4(1):1228.

17. Duff AJA. Psychological interventions in cystic fibrosis and asthma. Paediatric Respiratory Reviews. 2001;2(4):350-7. 
18. Hayes SC, Stroshal KD. A Practical Guide to Acceptance and Commitment Therapy. New York: Springer Science + Business Media; 2004.

19. Heffner M, Sperry J, Eifert GH, Detweiler M. Acceptance and commitment therapy in the treatment of an adolescent female with anorexia nervosa: A case example. Cognitive and Behavioral Practice. 2002;9(3):232-6.

20. Swain J, Hancock K, Dixon A, Koo S, Bowman J. Acceptance and Commitment Therapy for anxious children and adolescents: study protocol for a randomized controlled trial. Trials. 2013;14(1):140.

21. Wicksell RK, Dahl J, Magnusson B, Olsson GL. Using acceptance and commitment therapy in the rehabilitation of an adolescent female with chronic pain: A case example. Cognitive and Behavioral Practice. 2005;12(4):415-23.

22. Chong Y-y, Mak Y-w, Leung S-p, Lam S-y, Loke AY. Acceptance and Commitment Therapy for Parental Management of Childhood Asthma: An RCT. Pediatrics. 2019;143(2):e20181723.

23. Bahramiabdolmalaki S, Homayouni A, Aliyali M. Effectiveness of Acceptance and Commitment Therapy on Resilience, Psychological Well-Being, and Life Expectancy in Asthmatic Patients. The Journal of Tolooebehdasht. 2021;20(1).

24. Burns JM, Birrell E, Bismark M, Pirkis J, Davenport TA, Hickie IB, et al. The role of technology in Australian youth mental health reform. Australian health review : a publication of the Australian Hospital Association. 2016;40(5):584-90.

25. Andrews G, Bell C, Boyce P, Gale C, Lampe L, Marwat O, et al. Royal Australian and New Zealand College of Psychiatrists clinical practice guidelines for the treatment of panic disorder, social anxiety disorder and generalised anxiety disorder. 2018;52(12):1109-72.

26. Kim H, Xie B. Health literacy in the eHealth era: A systematic review of the literature. Patient Education and Counseling. 2017;100(6):1073-82.

27. RANZCP. Benefits of e-mental health treatments and interventions. Melbourne, Australia: The Royal Australian and New Zealand College of Psychiatrists; 2019. Report No.: Position statement 98 Contract No.: Position statement 98.

28. Boydell KM, Hodgins M, Pignatiello A, Teshima J, Edwards H, Willis D. Using technology to deliver mental health services to children and youth: a scoping review. Journal of the Canadian Academy of Child and Adolescent Psychiatry = Journal de l'Academie canadienne de psychiatrie de l'enfant et de l'adolescent. 2014;23(2):87-99.

29. Simister HD, Tkachuk GA, Shay BL, Vincent N, Pear JJ, Skrabek RQ. Randomized Controlled Trial of Online Acceptance and Commitment Therapy for Fibromyalgia. The Journal of Pain. 2018;19(7):741-53. 
30. Pennefather J, Hieneman M, Raulston TJ, Caraway N. Evaluation of an online training program to improve family routines, parental well-being, and the behavior of children with autism. Research in Autism Spectrum Disorders. 2018;54:21-6.

31. Bacca J, Baldiris S, Fabregat R, Graf S. Augmented reality trends in education: a systematic review of research and applications. 2014.

32. Akcayir M, Akcayir G. Advantages and challenges associated with augmented reality for education: A systematic review of the literature. Educational Research Review. 2017;20:1-11.

33. Porter ME, Heppelmann JE. How smart, connected products are transforming companies. Harvard Business Review. 2015;October(R1510G):1-19.

34. Dunleavy M, Dede C, Mitchell R. Affordances and Limitations of Immersive Participatory Augmented Reality Simulations for Teaching and Learning. Journal of Science Education and Technology. 2009;18(1):7-22.

35. Juan MC, Alcañiz Raya M, Monserrat C, Botella C, Baños R, Guerrero B. Using Augmented Reality to Treat Phobias. Computer Graphics and Applications, IEEE. 2005;25:31-7.

36. King CM, Carson KV, Van Agteren J, Kopsaftis ZA, Smith BJ, editors. A QUALITATIVE STUDY USING INNOVATIVE TECHNOLOGY VIA A SMARTPHONE APPLICATION TO PROVIDE DEMONSTRATIVE INHALER TECHNIQUE EDUCATION FOR ASTHMA PATIENTS. RESPIROLOGY; 2017: WILEY 111 RIVER ST, HOBOKEN 07030-5774, NJ USA.

37. Sharrad KJ, Kopsaftis ZA, Carson-Chahhoud KV, Stallman HM. The modifiable biopsychosocial drivers of psychological distress for adolescents with asthma: Implications for Clinical Care. Paediatr Respir Rev. 2021.

38. Sharrad K, Kopsaftis Z, Tai A, Spurrier N, Smith R, Esterman A, et al. Mixed Reality Technology as a Delivery Mechanism for Psychological Intervention in Adolescents With Asthma: A Qualitative Protocol. 2021;20:16094069211008333.

39. Toennesen LL, Soerensen ED, Hostrup M, Porsbjerg C, Bangsbo J, Backer V. Feasibility of highintensity training in asthma. European Clinical Respiratory Journal. 2018;5(1):1468714.

40. Yorke J, Adair P, Doyle A-M, Dubrow-Marshall L, Fleming S, Holmes L, et al. A randomised controlled feasibility trial of Group Cognitive Behavioural Therapy for people with severe asthma. Journal of Asthma. 2017;54(5):543-54.

41. Jama WMAJ. World Medical Association Declaration of Helsinki: ethical principles for medical research involving human subjects. 2013;310(20):2191-4. 


\section{Supplementary Files}

This is a list of supplementary files associated with this preprint. Click to download.

- Appendices.docx 